International Journal of Applied Mathematical Research, 6(1) (2017) 4-6
International Journal of Applied Mathematical Research
Sebsite: www.sciencepubco.com/index.php/IJAMR
doi: $10.14419 /$ ijamr.v6il.6875
Research paper

\title{
Implementation of multi-step differential transformation method for hyperchaotic Rossler system
}

\author{
Jafar Biazar $^{1 *}$, Tahereh Houlari ${ }^{1}$ and Roxana Asayesh ${ }^{1}$ \\ ${ }^{1}$ Department of Applied Mathematics, Faculty of Mathematical Sciences, University of Guilan,, P. O. Box 41335-1914, Guilan, Rasht, Iran \\ **Corresponding author E-mail: biazar@guilan.ac.ir
}

\begin{abstract}
In this work, the multi-step differential transformation method (MSDTM) is applied to approximate a solution of the hyperchaotic Rossler system. MSDTM is adapted from the differential transformation method (DTM). In this method, DTM is implemented in each subinterval. Results are compared with a fourth-order Runge Kutta method and a standard DTM. The results show that the MSDTM is an efficient and powerful technique for solving hyperchaotic Rossler systems and this method is more accurate than DTM.
\end{abstract}

Keywords: Hyperchaotic Rossler system; Differential transformation method; Forth-order Runge Kutta method; Multi-step differential transformation method.

\section{Introduction}

The behavior of dynamical systems such as circuits, mechanical devices, population growth, fluid dynamics, and weather are studied in a field of mathematics known as chaos theory. A chaotic system shows sensitivity to any tiny change in the initial conditions, so it has unpredictable behavior. In other words, it is impossible to predict the future behavior of a chaos system unless the initial conditions are entirely known and accurate. The first chaotic system, known as the Lorenz system, was discovered in 1963 by Lorenz for solving equations describing atmospheric flows [1]. Inspiring from Lorenz, Otto Rossler proposed a chaos system, known as Rossler system or Rossler attractor, resulted from studying a chemical reaction system[2]. This system contains three prototype first-order diferential equations with three dynamical variables in defining the phase space and three parameters. This system has been thoroughly studied by many researchers; (see [3, 4, 5] for more details). Continuing his work, In 1979 Rossler proposed another dynamical system which was made of four first order differential equations as following (see [6]).

Hyperchaotic Rossler system is the first four dimensional hyperchaotic system. This system contains four prototype ordinary differential equations as the following

$$
\left\{\begin{array}{l}
\frac{d x}{d t}=-y-z \\
\frac{d y}{d t}=x+a y+w \\
\frac{d z}{d t}=b+x z \\
\frac{d w}{d t}=c z+d w .
\end{array}\right.
$$

Where $x, y, z$, and w are state variables and $a, b, c$, and $d$ are positive parameters. It is well-known that this system exhibits hyperbolic behaviour when $a=0.25, b=3, c=-0.5$, and $d=0.05$. Here, with respect to these values of constants $a, b, c$, and $d$, Rossler system is studied by differential transformation method and multi-step differential transformation method in order to find its approximate solutions. see $[7,8,9]$ for more details. This paper is organized as follows: Section 2 presents some basic concepts of differential transformation method, facilitating the understanding of the ideas presented in this work. The multi-step differential transformation method and some important details pertaining to MSDTM will be addressed in section 3. Section 4 is devoted to showing the efficiency and the accuracy of the proposed method by applying MSDTM, R.K. and traditional DTM on the Rossler system and the results are presented in some tables. So, the results can be compared. Some conclusions are drawn in section 5 .

\section{Differential transformation method}

the basic idea of differential transform method (DTM) is addressed in many papers such as $[2,7]$. So, knowing the important properties of this method which are implemented to achieve our goals are outlined in this section. Let

$Y(k)=\frac{1}{k !}\left[\frac{d^{k} y(k)}{d x^{k}}\right]_{x=0}$,

where $y(x)$ is the solution of differential equation and $Y(k)$ is the differential transformed of $y(k)$. Differentian inverse transformation of $Y(k)$ is defined as follows

$y(x)=\sum_{k=0}^{\infty} x^{k} Y(k)$

Therefore, by combining (2) and (3), the following equation will be obtained.

$y(x)=\sum_{k=0}^{\infty}\left[\frac{d^{k} y(k)}{d x^{k}}\right]_{x=0} \frac{x^{k}}{k !}$. 
In fact, the concept of differential transformation is gaind from the Tylor series expansion. In order to understand how this method is implemented in Rossler system, it's enough to know some essential properties of DTM [9].

1) If $f(x)=g(x) \pm h(x)$, then $F(K)=G(k) \pm H(k)$,

2) If $f(x)=\alpha g(x)$, then $F(k)=\alpha G(k)$,

3) If $f(x)=g(x) h(x)$, then $F(k)=\sum_{l=0}^{k} G(l) H(k-l)$,

4) If $f(x)=\left(x-x_{0}\right)^{p}$, then $F(k)=\delta(k-p)$ where

$\delta(k)= \begin{cases}1 & \text { if } k=0, \\ 0 & \text { if } k \neq 0,\end{cases}$

5) If $f(x)=\frac{d^{m} g(x)}{d x^{m}}$, then $F(k)=(k+1)(k+2) \cdots(k+m) G(k+m)$.

\section{Multistep Differential Transform Method}

According to the knowledge one learns from section 2, DTM determines a series solution,by a recurrence relationship, over the whole domain. This approximate solution is valid in the neightborhood of a fixed point $t_{0}$. Therefore, the series solution always converges in a small region and this is a drawback of DTM. In order to enhance the efficiency of DTM,the idea of MSDTM is introduced. see [7] for more details and examples.

Consider the following system of differential equations

$$
\begin{aligned}
& D y_{1}(t)=f_{1}\left(t, y_{1}, y_{2}, \cdots, y_{n}\right), \\
& D y_{2}(t)=f_{2}\left(t, y_{1}, y_{2}, \cdots, y_{n}\right), \\
& \vdots \\
& D y_{n}(t)=f_{n}\left(t, y_{1}, y_{2}, \cdots, y_{n}\right),
\end{aligned}
$$

subject to the following initial conditions

$y_{i}(t)=L_{i}, \quad i=1,2, \cdots, n$.

Where $\mathrm{D}$ is the first order differentiation operator. Let $[0, T]$ be the whole interval in which the solution of considered initial value problem (5)-(6) is going to be find. In the multi-step differential transform method (DTM), the initial interval $[0, T]$ is divided into $M$ subintervals $\left[t_{m-1}, t_{m}\right]$, of equal step size $h=\frac{T}{M}$, by using the nodes $t_{m}=m h$, $m=1,2, \cdots, M$. After that, the DTM will be applied in each subinterval. For example, in the first step, the DTM is applied to the initial value problem (5)-(6) over the first subinterval $\left[0, t_{1}\right]$. Then, using the initial condition $y_{i}(0)=L_{i}$, for $i=1,2, \cdots, n$, the approximate solution $y_{i-1}(t), t \in\left[0, t_{1}\right]$ will be found. For $m \geq 2$ and at each subinterval $\left[t_{m-1}, t_{m}\right]$, initial condition $y_{i, m}\left(t_{m-1}\right)=y_{i, m-1}\left(t_{m-1}\right)$ will be used. This process is repeated and it will generate a sequence of approximate solutions $y_{i, m}(t), m=1,2, \cdots, M$, for $i=1,2, \cdots, n$. So, the MSDTM finds the solution as follows

$y_{i}(t)= \begin{cases}y_{i, 1}(t), & t \in\left[t_{0}, t_{1}\right] \\ y_{i, 2}(t), & t \in\left[t_{1}, t_{2}\right] \\ \vdots & \\ y_{i, m}(t), & t \in\left[t_{m-1}, t_{m}\right] .\end{cases}$

\section{Application}

Applying the differential transformation method to the hyperchaotic Rossler system (1) gives

$$
\left\{\begin{array}{l}
X(k+1)=\frac{1}{k+1}[-Y(k)-Z(k)] \\
Y(k+1)=\frac{1}{k+1}[X(k)+a Y(k)+W(k)] \\
Z(k+1)=\frac{1}{k+1}\left[b+\sum_{l=0}^{k} X(l) Z(k-l)\right] \\
W(k+1)=\frac{1}{k+1}[-c Z(k)+d W(k)]
\end{array}\right.
$$

where $X(k), Y(k), Z(k)$, and $W(k)$ are the differential transformations of the corresponding functions $x(t), y(t), z(t)$, and $w(t)$, respectively. The initial conditions are given by

$X(0)=-20, \quad Y(0)=0, \quad Z(0)=0, \quad W(0)=15$.

Applying the pertinent inverse differential transformation procedure, the solutions will be as follows.

$$
\begin{aligned}
& x(t)=1407648726378237 t^{9} / 128000000000 \\
& -1572638143690213 t^{8} / 327680000000 \\
& +2487210665898667 t^{7} / 1310720000000 \\
& -5553725570125369 t^{6} / 8388608000000 \\
& +5331126534362453 t^{5} / 26843545600000-159657 t^{4} / 3200 \\
& +5529627228023467 t^{3} / 549755813888000+t^{2}-20 \\
& y(t)=-6475229820351271 t^{9} / 8192000000000 \\
& +4607966807557827 t^{8} / 13107200000000 \\
& -1469836042426433 t^{7} / 10485760000000 \\
& +6584185673919147 t^{6} / 134217728000000 \\
& -3170877997492907 t^{5} / 214748364800000 \\
& +5171426955537067 t^{4} / 1374389534720000 \\
& +11 t^{3} / 160-t^{2} / 4-5 t \\
& z(t)=3783405969482637 t^{9} / 16000000000 \\
& -1271383821325919 t^{8} / 12800000000 \\
& +1262703752584753 t^{7} / 32768000000 \\
& -3494954669902507 t^{6} / 262144000000 \\
& +3189679703 t^{5} / 800000-31896397 t^{4} / 32000 \\
& +79801 t^{3} / 400-1197 t^{2} / 40+3 t \\
& w(t)=5636927324223121 t^{9} / 1024000000000 \\
& -7872443841834809 t^{8} / 3276800000000 \\
& +2490190312946841 t^{7} / 2621440000000 \\
& -1112093667447057 t^{6} / 3355443200000 \\
& +2545343601 t^{5} / 25600000-6368159 t^{4} / 256000 \\
& +15921 t^{3} / 3200-117 t^{2} / 160+3 t / 4+15
\end{aligned}
$$

Implementation MSDTM results in a polynomial approximation for each state variables, in different subintervals.

$$
\begin{aligned}
& x(t)=10497.4806 t^{9}-4587.9262 t^{8}+1815.09204 t^{7} \\
& -633.4187 t^{6}+189.9830 t^{5}+47.7671 t^{4}+9.5833 t^{3}+t^{2}-20 \\
& y(t)=-755.6137 t^{9}+336.2742 t^{8}-134.1117 t^{7}+46.9267 t^{6} \\
& -14.1373 t^{5}+3.5845 t^{4}+0.0678 t^{3} \quad t \in[0,0.01] \\
& -0.2500 t^{2}-5 t \\
& z(t)=2.2504 t^{9}-94813.6001 t^{8}+36837.5218 t^{7}-12752.5710 t^{6} \\
& +3814.6500 t^{5}-953.5000 t^{4}+191 t^{3}-\frac{57}{2} t^{2}+3 t \\
& w(t)=5254.6629 t^{9}-2296.6661 t^{8}+908.6329 t^{7}-317.0949 t^{6} \\
& +95.1118 t^{5}-23.8157 t^{4}+4.7378 t^{3}-0.7312 t^{2}+0.75 t+15
\end{aligned}
$$


Table 1: Table 1. Differences between 10-term DTM and RK4 solutions

\begin{tabular}{ccccc}
\hline$t$ & \multicolumn{4}{c}{$\Delta=|D T M-R K 4|$} \\
\hline & $\triangle x$ & $\Delta y$ & $\triangle z$ & $\Delta w$ \\
\hline 1 & $4.6081 \times 10^{6}$ & $3.2992 \times 10^{5}$ & $9.9834 \times 10^{7}$ & $2.3066 \times 10^{6}$ \\
2 & $4.6081 \times 10^{6}$ & $3.2992 \times 10^{5}$ & $9.9834 \times 10^{7}$ & $2.3066 \times 10^{6}$ \\
3 & $1.8868 \times 10^{8}$ & $1.3526 \times 10^{7}$ & $4.0781 \times 10^{9}$ & $9.4447 \times 10^{7}$ \\
4 & $2.5969 \times 10^{9}$ & $1.8628 \times 10^{8}$ & $5.6058 \times 10^{10}$ & $1.2999 \times 10^{9}$ \\
5 & $1.9743 \times 10^{10}$ & $1.4167 \times 10^{9}$ & $4.2586 \times 10^{11}$ & $9.8824 \times 10^{9}$ \\
6 & $1.0327 \times 10^{11}$ & $7.4123 \times 10^{9}$ & $2.2264 \times 10^{12}$ & $5.1692 \times 10^{10}$ \\
7 & $4.1760 \times 10^{11}$ & $2.9980 \times 10^{10}$ & $8.9998 \times 10^{12}$ & $2.0903 \times 10^{11}$ \\
8 & $1.3993 \times 10^{12}$ & $1.0047 \times 10^{11}$ & $3.0149 \times 10^{13}$ & $7.0044 \times 10^{11}$ \\
9 & $4.06279 \times 10^{12}$ & $2.9174 \times 10^{11}$ & $8.7512 \times 10^{13}$ & $2.0336 \times 10^{12}$ \\
10 & $1.0536 \times 10^{13}$ & $7.5663 \times 10^{11}$ & $2.2690 \times 10^{14}$ & $5.2737 \times 10^{12}$ \\
\hline
\end{tabular}

Table 2: Table 2. Differences between 10-term MSDTM and RK4 solutions

\begin{tabular}{|c|c|c|c|c|}
\hline \multirow[t]{2}{*}{$t$} & \multicolumn{3}{|c|}{$\triangle=|M S D T M-R K 4|$} & \multirow[b]{2}{*}{$\triangle w$} \\
\hline & $\triangle x$ & $\triangle y$ & $\triangle z$ & \\
\hline 1 & 0.0007 & 0.0003 & 0.0008 & 0.0002 \\
\hline 2 & 0.0007 & 0.0018 & 0.0012 & 0.0007 \\
\hline 3 & 0.0007 & 0.0036 & 0.0015 & 0.0014 \\
\hline 4 & 0.0035 & 0.0046 & 0.0012 & 0.0025 \\
\hline 5 & 0.0069 & 0.0034 & 0.0007 & 0.0034 \\
\hline 6 & 0.0080 & 0.0001 & 0.0006 & 0.0041 \\
\hline 7 & 0.0056 & 0.0033 & 0.0006 & 0.0047 \\
\hline 8 & 0.0016 & 0.0026 & 0.0009 & 0.0054 \\
\hline 9 & 0.0001 & 0.0026 & 0.0017 & 0.0062 \\
\hline 10 & 0.0044 & 0.0093 & 0.0016 & 0.0076 \\
\hline
\end{tabular}

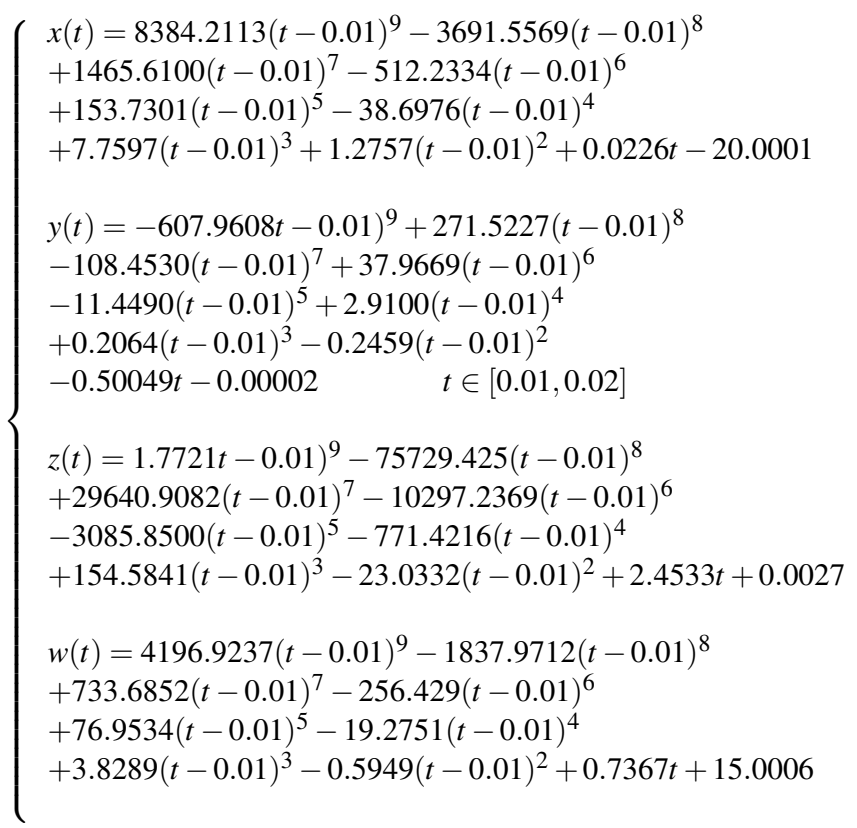

where $X_{i}(n), Y_{i}(n), Z_{i}(n)$ and $W_{i}(n)$ for $i=1,2, \cdots, m$ satisfy the recurrence relations in system (1) subject to the initial conditions $X_{i}(0)=x_{i}\left(t_{i-1}\right)=x_{i-1}\left(t_{i-1}\right), Y_{i}(0)=y_{i}\left(t_{i-1}\right)=y_{i-1}\left(t_{i-1}\right)$, $Z_{i}(0)=z_{i}\left(t_{i-1}\right)=z_{i-1}\left(t_{i-1}\right)$ and $W_{i}(0)=w_{i}\left(t_{i-1}\right)=w_{i-1}\left(t_{i-1}\right)$.

In the tables 1 and 2 , the absolute error between 10-term DTM solutions and MSDTM solutions with time step $\triangle t=0.01$ and the RK4 solutions with $\triangle t=0.01$, are presented, respectively. Results are presented up to $t=10$, in ten integer points. Comparisons show that MSDTM is more accurate than DTM as compared to RK4.

\section{Conclutions}

In this paper, both differential transformation method (DTM) and multi-step differential transformation method (MSDTM) have been succesfully applied to compute an approximated solution of hyperchaotic Rossler system. In spite of requiring more computations in MSDTM, because DTM is applied on a thousand subintervals,but more accurate results are obtained. These results also show that the approximate solutions obtained by MSDTM are valid for a long time interval, while DTM fails to achieve this accuracy.

\section{References}

[1] E. N. Lorenz, "Deterministic non-periodic flow", Journal Of the Atmospheric Sciences, Vol.20, (1963), p.p 130-141.

[2] O. E. Rössler, "An equation for continuous chaos", Physics Letters A,Vol. 57, (1976), p.p 397-398

[3] Dimitris T. Maris, Dimitris A. Goussis, "The "hidden" dynamics of the Rossler attractor", Physica D: Nonlinear Phenomena, Vol. 295-296, (2015), p.p 66-90.

[4] R. Barrio, F. Blesa, A. Dena, S. Serrano, "Qualitative and numerical analysis of the Rossler model: Bifurcations of equilibria", Computers Mathematics with Applications, Vol. 62, Issue 11, (2011), p.p 41404150.

[5] R. Barrio, F. Blesa, S. Serrano, "Qualitative analysis of the Rössler equations: Bifurcations of limit cycles and chaotic attractors", Physica D: Nonlinear Phenomena, Vol. 238, Issue 13, (2009) p.p 1087-1100.

[6] O. Rössler, "An equation for hyper chaoti”, Phys Lett A, Vol. 71, (1979), p.p $155-7$.

[7] E. Abuteen, S. Momani, A. Alawneh, "Solving the fractional nonlinear Bloch system multi-step generalized differential transform", Computers Mathematics with Applications, Vol. 68, Issue 12, Part A, (2014), p.p 2124-2132.

[8] A. Arikoglu, I. Ozkol, "Solution of fractional dierential equations by using dierential transform method", Chaos, Solitons and Fractals, Vol 34, (2007), p.p 1473-1481.

[9] M. El-Gamel, M. Abd El-Hady, "Computation of the Eigenvalues and Eigenfunction of Generalized Sturm-Liouville Problems via the Differential Transformation Method", IJRRAS, Vol. 15, (2013), p.p 259-268. 